\title{
TITLE:
}

\section{Linear and nonlinear optical properties of an autoionizing medium}

$\operatorname{AUTHOR}(S)$ :

Nakajima, T

CITATION:

Nakajima, T. Linear and nonlinear optical properties of an autoionizing medium. PHYSICAL REVIEW A 2001, 63(4): 043804.

ISSUE DATE:

2001-04

URL:

http://hdl.handle.net/2433/50413

RIGHT:

Copyright 2001 American Physical Society 
PHYSICAL REVIEW A, VOLUME 63, 043804

\title{
Linear and nonlinear optical properties of an autoionizing medium
}

\author{
Takashi Nakajima \\ Institute of Advanced Energy, Kyoto University, Gokasho, Uji, Kyoto 611-0011, Japan
}

(Received 31 August 2000; published 7 March 2001)

\begin{abstract}
We study the linear and nonlinear optical properties of autoionizing systems under the action of a singlelaser field. It is found that the group velocity is greatly reduced by the presence of a neighboring autoionizing state. Conditions for self-focusing or defocusing are also derived for the case of an isolated autoionizing system.
\end{abstract}

DOI: 10.1103/PhysRevA.63.043804

PACS number(s): 42.50.Gy, 42.65.An, 32.80.Dz, 42.65.-k

\section{INTRODUCTION}

During the past several years extensive studies have been carried out for understandings/applications of systems exhibiting electromagnetically induced transparency (EIT) [1-6]. The essence of the EIT is to make use of a dressing laser for modifying the absorptive as well as dispersive properties of the medium. Most recently extreme reduction of light speed down to $17 \mathrm{~m} / \mathrm{s}$ has been observed in an ultracold atomic gas utilizing the EIT scheme [5]. It should be noted that the light speed reduction could be still significant, down to $90 \mathrm{~m} / \mathrm{s}$, even with hot atomic vapor as a medium, as demonstrated by Kash et al. [6]. In both experiments alkali-metal atoms have been employed because of the existence of the almost ideal metastable states, i.e., hyperfine sublevels of the ground state.

It is well known that a photoabsorption or photoionization profile into the vicinity of an autoionizing state exhibits a minimum, and it can reach zero if the autoionizing state consists of one discrete and one continuum states only. In contrast to the EIT scheme, transparency of an autoionizing scheme can be realized without a dressing laser due to the intra-atomic interaction or configuration interaction. In terms of the dressed-state picture, it can be understood that the discrete component of an autoionizing state is "dressed" by the continuum component via intra-atomic interaction that is purely nonradiative, leading to the constructive or destructive interference, while an intermediate bound state of the EIT scheme is dressed by the third bound state via dipole interaction. Obviously nonradiative or radiative coherence plays an essential role in both cases.

Knowing the similarity between these two schemes, it might come to one's mind that radiative coherence, which is necessary for various coherence-related phenomena, might be replaced by nonradiative coherence. Harris and his coworkers have demonstrated that the nonlinear susceptibility can be enhanced while canceling linear absorption by tuning a laser to the absorption window between two autoionizing lines [7], where the underlying idea is similar to that of the EIT scheme, as demonstrated by the same group [8].

Recently we have shown that an enhancement of Kerrtype nonlinearity, which was originally proposed by Schmidt and Imamoğlu for a four-level system interacting with threecolor lasers [9], could be observed for a quasi-three-level autoionizing system interacting with two-color lasers [10]. If the autoionizing system could be used as a dispersive or nonlinear medium, it might be useful, particularly in the VUV-XUV wavelength region, since an autoionizing state necessarily lies above the ionization threshold and the transition wavelength from the ground state is typically shorter than $200 \mathrm{~nm}$. Furthermore, in some cases the use of the autoionizing system leads to the reduction of the number of lasers needed [10], which could be of great advantage because of the experimental simplification. Closely related to this work, we note that the dispersive properties of an EIT system have been first studied by Harris and his co-workers [3], and the linear and nonlinear response of various bound state systems by Scully and his co-workers [11,12]. Optical properties of autoionizing systems have also been studied in terms of lasing without inversion $[13,14]$ and efficient sumfrequency generation via absorption window between two autoionizing states [7].

In this paper we study the linear and nonlinear optical properties of an autoionizing medium under the action of a single laser field. Especially we focus on the variation of the group velocity at the absorption minimum for an isolated and a double-autoionizing system. In addition, we investigate the conditions for self-focusing/-defocusing for the case of an isolated-autoionizing system. For a theoretical description we employ a set of density-matrix equations. After deriving necessary equations some representative numerical results are shown for illustration.

\section{MODEL}

\section{A. Basic equations}

The system we consider consists of one bound (usually the ground) state $|1\rangle$ and two neighboring excited states $|2\rangle$ and $|3\rangle$ embedded in a common continuum $|c\rangle$. The discrete states $|2\rangle$ and $|3\rangle$ are coupled to the continuum by configuration interaction $V$ and hence autoionize. Under the influence of a single-laser field tuned near resonance, the time evolution of this system is described by the following set of density-matrix equations $[15,16]$,

$$
\begin{aligned}
& \dot{\sigma}_{11}=-\gamma_{1} \sigma_{11}+2 \operatorname{Im}\left(\widetilde{\Omega}_{2} \sigma_{21}\right)+2 \operatorname{Im}\left(\widetilde{\Omega}_{3} \sigma_{31}\right), \\
& \dot{\sigma}_{22}=-\Gamma_{2} \sigma_{22}-2 \operatorname{Im}\left(\widetilde{\Omega}_{2}^{*} \sigma_{21}\right)-2 \operatorname{Im}\left(\widetilde{\Omega}_{32}^{*} \sigma_{23}\right), \\
& \dot{\sigma}_{33}=-\Gamma_{3} \sigma_{33}-2 \operatorname{Im}\left(\widetilde{\Omega}_{3}^{*} \sigma_{31}\right)-2 \operatorname{Im}\left(\widetilde{\Omega}_{32}^{*} \sigma_{32}\right),
\end{aligned}
$$




$$
\begin{gathered}
\dot{\sigma}_{21}=\left[i \delta_{2}-\frac{1}{2}\left(\gamma_{1}+\Gamma_{2}\right)\right] \sigma_{21}-i \widetilde{\Omega}_{2} \sigma_{11}+i \widetilde{\Omega}_{2}^{*} \sigma_{22}-i \widetilde{\Omega}_{3}^{*} \sigma_{23}, \\
\dot{\sigma}_{31}=\left[i \delta_{3}-\frac{1}{2}\left(\gamma_{1}+\Gamma_{3}\right)\right] \sigma_{31}-i \widetilde{\Omega}_{3} \sigma_{11}+i \widetilde{\Omega}_{3}^{*} \sigma_{33}-i \widetilde{\Omega}_{2}^{*} \sigma_{32}, \\
\dot{\sigma}_{32}=\left[i\left(\delta_{3}-\delta_{2}\right)-\frac{1}{2}\left(\Gamma_{2}+\Gamma_{3}\right)\right] \sigma_{32}-i \widetilde{\Omega}_{3} \sigma_{12}+i \widetilde{\Omega}_{2}^{*} \sigma_{31},
\end{gathered}
$$

where $\gamma_{1}$ is the direct ionization width of the lower state $|1\rangle$, while $\Gamma_{2}$ and $\Gamma_{3}$ are the autoionization widths of states $|2\rangle$ and $|3\rangle$, respectively. The detunings are defined as $\delta_{j}=\left(E_{j}\right.$ $\left.-E_{1}\right)-\hbar \omega(j=2,3)$ with $\omega$ being the laser frequency and $E_{k}(k=1,2,3)$ the energy of state $|k\rangle$. Complex Rabi frequencies $\widetilde{\Omega}_{j}(j=2,3)$ are defined as

$$
\widetilde{\Omega}_{j}=\Omega_{j}\left(1-\frac{i}{q_{j}}\right) \quad(j=2,3) .
$$

The nonradiative coupling between the two autoionizing states through the continuum is represented by the complex quantity $\widetilde{\Omega}_{32}$, which is defined as

$$
\widetilde{\Omega}_{32}=\mathrm{P} \int d E_{c} \frac{V_{2 c} V_{c 3}}{E_{1}+\hbar \omega-E_{c}}-\frac{i}{2} \sqrt{\Gamma_{2} \Gamma_{3}},
$$

where $\mathrm{P}$ denotes the principal-value part of the integral. The real part of Eq. (8) looks similar to the expression for Raman-type radiative coupling. However, their origins are physically different in a sense that for the case of the Ramantype coupling, the coupling is caused by the external electromagnetic field, while in this case it is caused by the intraatomic electrostatic interaction represented by $V_{2 c}$ and $V_{c 3}$ through the continuum. Naturally this kind of term does not exist between two bound states, or two discrete states lying in the continua but not decaying to the common continuum.

\section{B. Linear susceptibility}

Once the above set of density-matrix equations has been solved, the induced polarization $P_{\omega}$ can be computed from the following relation,

$$
P_{\omega}=\left(\mu_{12} \sigma_{21}+\mu_{13} \sigma_{31}+\sum_{c} \mu_{1 c} \sigma_{c 1}\right)+\text { c.c., }
$$

where $\sigma_{c 1}$ is an off-diagonal density-matrix element for $|1\rangle$ and $|c\rangle$ that has already been eliminated when deriving Eqs. (1)-(6) and $\mu_{i j}$ is a dipole-matrix element between states $|i\rangle$ and $|j\rangle$. Under the weak-pumping condition, Eqs. (1)-(6) can be easily solved, and by substituting the solution into Eq. (9), we obtain an expression of the linear susceptibility $\chi^{(1)}$ as

$$
\chi^{(1)}=\frac{N}{\epsilon_{0} \hbar}\left[-\left(s_{1}^{\prime}-i \frac{\gamma_{1}^{\prime}}{2}\right)-\frac{\tilde{\mu}_{12}^{2}\left[\delta_{3}+\frac{i}{2}\left(\gamma_{1}+\Gamma_{2}\right)\right]+\tilde{\mu}_{13}^{2}\left[\delta_{2}+\frac{i}{2}\left(\gamma_{1}+\Gamma_{2}\right)\right]+2 \tilde{\mu}_{12} \tilde{\mu}_{13} \widetilde{\Omega}_{32}}{\left[\delta_{2}+\frac{i}{2}\left(\gamma_{1}+\Gamma_{2}\right)\right]\left[\delta_{3}+\frac{i}{2}\left(\gamma_{1}+\Gamma_{3}\right)\right]-\left(\widetilde{\Omega}_{32}\right)^{2}}\right],
$$

where $\tilde{\mu}_{1 k}=\widetilde{\Omega}_{k} / \varepsilon(k=2,3), s_{1}^{\prime}=s_{1} / \varepsilon$, and $\gamma_{1}^{\prime}=\gamma_{1} / \varepsilon$ with $\varepsilon$ being the field amplitude, $s_{1}$ the level shift of $|1\rangle$ due to the coupling with $|c\rangle$ via $V$, and $N$ the atomic density. It should be noted that the interference term $2 \tilde{\mu}_{12} \tilde{\mu}_{13} \widetilde{\Omega}_{32}$ appears in the above equation. In particular, if $\operatorname{Re}\left(\Omega_{32}\right) \ll \operatorname{Im}\left(\Omega_{32}\right)$ that is usually the case, Eq. (10) can be reduced to the form of

$$
\chi^{(1)}=\frac{N}{\epsilon_{0} \hbar}\left[-\left(s_{1}^{\prime}-i \frac{\gamma_{1}^{\prime}}{2}\right)-\frac{\tilde{\mu}_{12}^{2}\left(\delta_{3}+\frac{i}{2} \Gamma_{3}\right)+\tilde{\mu}_{13}^{2}\left(\delta_{2}+\frac{i}{2} \Gamma_{2}\right)-i \tilde{\mu}_{12} \tilde{\mu}_{13} \sqrt{\Gamma_{2} \Gamma_{3}}}{\delta_{2} \delta_{3}+i\left(\delta_{2} \frac{\Gamma_{3}}{2}+\delta_{3} \frac{\Gamma_{2}}{2}\right)}\right] .
$$

Alternatively, the real and imaginary parts of Eq. (11) can be written, in a dimensionless form, as

$$
\begin{aligned}
\operatorname{Re}\left(\chi^{(1)}\right)= & \frac{N}{\epsilon_{0} \hbar}\left\{-s_{1}^{\prime}-\frac{\gamma_{1}^{\prime}}{2} \frac{1}{\left(\epsilon_{2} \epsilon_{3}\right)^{2}+\left(\epsilon_{2}+\epsilon_{3}\right)^{2}}\right. \\
& \times\left[\epsilon_{2}\left(q_{2} \epsilon_{3}+q_{3}\right)^{2}+\epsilon_{3}\left(q_{2}+q_{3} \epsilon_{2}\right)^{2}\right. \\
& -2 \epsilon_{2} \epsilon_{3}\left(2 q_{2} q_{3}+\epsilon_{2}+\epsilon_{3}\right)+\epsilon_{2}\left(q_{2}-\epsilon_{3}\right)^{2}
\end{aligned}
$$

$$
\begin{aligned}
& +\epsilon_{3}\left(q_{3}-\epsilon_{2}\right)^{2}-2 q_{2} \epsilon_{3}\left(q_{3}+\epsilon_{3}\right) \\
& \left.\left.-2 q_{3} \epsilon_{2}\left(q_{2}+\epsilon_{2}\right)\right]\right\}
\end{aligned}
$$

and

$$
\operatorname{Im}\left(\chi^{(1)}\right)=\frac{N}{\epsilon_{0} \hbar}\left[\frac{\gamma_{1}^{\prime}}{2} \frac{\left(q_{2} \epsilon_{3}+q_{3} \epsilon_{2}+\epsilon_{2} \epsilon_{3}\right)^{2}}{\left(\epsilon_{2} \epsilon_{3}\right)^{2}+\left(\epsilon_{2}+\epsilon_{3}\right)^{2}}\right],
$$


TABLE I. Linear susceptibilities and derivatives of the isolatedautoionizing system. All quantities are to be multiplied by $N / \epsilon_{0} \hbar$.

\begin{tabular}{lccc}
\hline \hline$\chi^{(1)}$ & $\frac{\partial \chi^{(1)}}{\partial \omega}$ & $\frac{\partial^{2} \chi^{(1)}}{\partial \omega^{2}}$ \\
\hline Real part & $-s_{1}^{\prime}+\frac{2 \mu_{12}^{2}}{q_{2} \Gamma_{2}}$ & $\frac{4 \mu_{12}^{2}}{q_{2}^{2} \Gamma_{2}^{2}}$ & $\frac{16 \mu_{12}^{2}}{q_{2}\left(q_{2}^{2}+1\right) \Gamma_{2}^{3}}$ \\
Imaginary part & 0 & 0 & $\frac{16 \mu_{12}^{2}}{q_{2}^{2}\left(q_{2}^{2}+1\right) \Gamma_{2}^{3}}$ \\
\hline \hline
\end{tabular}

where dimensionless detunings are defined as $\epsilon_{k}$ $=\delta_{k} /\left(\Gamma_{k} / 2\right)(k=2,3)$. Using Eqs. (12) and (13), analytical expressions of the dispersion terms can be derived, but they are too complicated to extract any physical picture. Therefore we do not dare to present them here. However, if the upper state consists of an isolated-autoionizing state, i.e., $\mu_{13}=0$, dispersion terms can be written in a compact form, and their expressions at the absorption minimum located at $\delta_{2}=-q_{2} \Gamma_{2} / 2$ are presented in Table I. The group velocity can be calculated using the following formula:

$$
\frac{1}{v_{g}}=\frac{1}{c}+\frac{\pi}{\lambda} \frac{\partial \chi^{(1)}}{\partial \omega}
$$

where $c$ is the speed of light in vacuum and $\lambda$ is the wavelength of the incident laser. Since the dispersion $\partial \chi^{(1)} / \partial \omega$ at the absorption minimum is always positive for any $q_{2}$, the corresponding group velocity is always smaller than that in vacuum. In many cases it is more convenient to rewrite the dispersion term in terms of the ionization cross section $\sigma_{0}(E)$ into the smooth continuum and $\Gamma_{2}$ rather than $\mu_{12}$, $q_{2}$, and $\Gamma_{2}$, since without any resonant state nearby, $\sigma_{0}(E)$ is usually a slowly varying function of continuum energy $E$, and it is experimentally/theoretically easier to obtain than $\mu_{12}$ and $q_{2}$. When the quantities $\mu_{12}, \Gamma_{2}$, and $\sigma_{0}(E)$ are given in atomic units, we find that they are connected by the relation,

$$
\left|\mu_{12}\right|^{2}=\frac{\pi}{2} q_{2}^{2} \Gamma_{2} \sigma_{0}(E)
$$

and $\partial \chi^{(1)} / \partial \omega$ at the absorption minimum can be recast into the form as

$$
\frac{\partial \chi^{(1)}}{\partial \omega}=2 \pi \frac{\sigma_{0}}{\Gamma_{2}}
$$

which is independent of $q_{2}$ once $\sigma_{0}$ and $\Gamma_{2}$ are given. Recalling that $\sigma_{0}$ does not vary so much within the energy range of $1 \mathrm{eV}$ or so, and $\Gamma_{2}$ is roughly proportional to $1 / \mathrm{n}^{3}$ for an $n^{\prime} l^{\prime} n l$ autoionizing Rydberg state converging to the ionic $n^{\prime} l^{\prime}$ state, $\operatorname{Re}\left(\partial \chi^{(1)} / \partial \omega\right)$ can be larger for larger $n$, in principle, for any elements. As for the group velocity dispersion term $\partial^{2} \chi^{(1)} / \partial \omega^{2}$ in Table I, it can be positive or negative at the absorption minimum, depending on the sign of $q_{2}$.
These general results should be compared with those for the EIT scheme obtained by Harris et al. [3].

\section{Nonlinear susceptibility}

In order to examine the self-focusing/-defocusing effect of an autoionizing medium, we now need to derive an expression for the third-order index of refraction. For simplicity we consider the case of an isolated-autoionizing state only, i.e., $\mu_{13}=0$. Adding to Eqs. (1) - (6) the repump terms $\lambda_{2}$ and $\lambda_{i}$ from states $|2\rangle$ and $|c\rangle$ back to the lower state $|1\rangle$, respectively, and assuming a steady state, the third-order susceptibility can be derived, after some algebra, as [10]

$$
\begin{aligned}
\chi^{(3)}= & \frac{N}{\epsilon_{0} \hbar^{3}}\left[\frac{-\mu_{12}^{2} \varepsilon \sigma_{11}^{(2)}+\left|\mu_{12}\right|^{2} \varepsilon \sigma_{22}^{(2)}-\frac{i}{2} \gamma_{1}^{\prime} \varepsilon^{2} \sigma_{21}^{(1)}}{\delta_{2}+\frac{i}{2}\left(\lambda_{2}+\Gamma_{2}\right)}\right. \\
& \left.-\left(s_{1}^{\prime}-i \frac{\gamma_{1}^{\prime}}{2}\right) \varepsilon \sigma_{11}^{(2)}\right] .
\end{aligned}
$$

Using this equation the index of refraction can be expanded as a power series of field amplitude $\varepsilon$ as

$$
n(\varepsilon)=n_{0}+n_{2}|\varepsilon|^{2}+\cdots=n_{0}+\frac{\chi^{(3)}}{2 n_{0}}|\varepsilon|^{2}+\cdots,
$$

with $n_{0}$ and $n_{2}$ being the linear and third-order indices of refraction defined by $n_{0}^{2}=1+\chi^{(1)}$ and $n_{2}=\chi^{(3)} / 2 n_{0}$. Obviously self-focusing will occur when $\chi^{(3)}>0$. At the absorption minimum $\delta_{2}=-q_{2} \Gamma_{2} / 2$, the third-order susceptibility given in Eq. (17) can be simplified to be

$$
\chi^{(3)}=\frac{N}{\epsilon_{0} \hbar^{3}}\left\{\frac{2 \mu_{12}^{2}}{\left(q_{2} \Gamma_{2}\right)^{2}}\left[\frac{-4 \mu_{12}^{2}}{q_{2} \Gamma_{2}}\left(2-\frac{\lambda_{2}}{\lambda_{i}}\right)+2 s_{1}^{\prime}\left(1-\frac{\lambda_{2}}{\lambda_{i}}\right)\right]\right\},
$$

which is pure real, and its sign can be positive or negative, depending on the sign of $q_{2}$ and the values of repump rates $\lambda_{2}$ and $\lambda_{i}$, and $s_{1}^{\prime}$. Therefore, whether a self-focusing or -defocusing occurs via absorption minimum must be carefully examined case by case.

\section{NUMERICAL EXAMPLES}

In this section, we present a few numerical examples for illustration. Although we have no specific atoms in mind, we have chosen the values of parameters in a realistic way in that the orders of parameters chosen here are typical for most of the metal elements.

In Fig. 1 we show representative profiles of the real and imaginary parts of the linear susceptibility $\chi^{(1)}$ and its derivative $\partial \chi^{(1)} / \partial \omega$ as a function of normalized detuning $\epsilon_{2}$ for an isolated autoionizing system. In this example we have chosen the asymmetry parameter to be $q_{2}=3$. As pointed out 

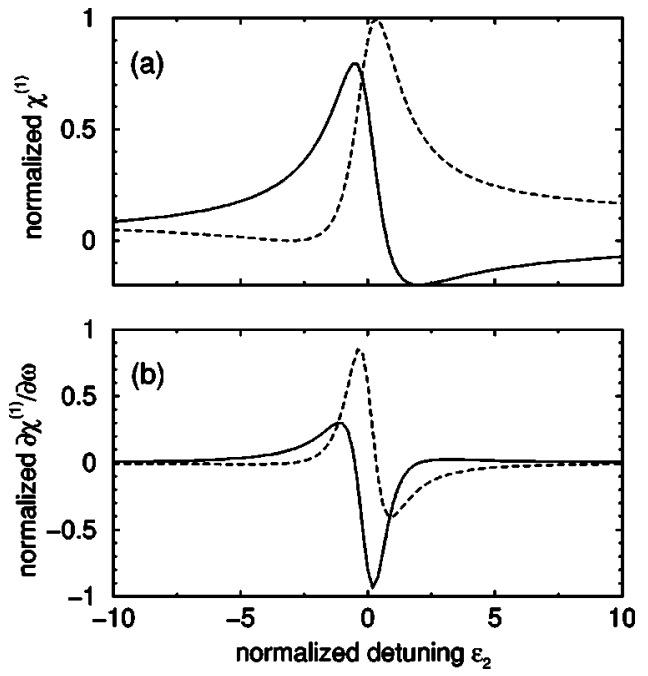

FIG. 1. (a) Real and imaginary parts of the linear susceptibility $\chi^{(1)}$ for the isolated-autoionizing system as a function of normalized detuning $\epsilon_{2}$. (b) Real and imaginary parts of $\partial \chi^{(1)} / \partial \omega$. Normalization is to the peak value of the imaginary part of $\chi^{(1)}$.

in Ref. [16], a large linear index of refraction can be obtained while canceling absorption at the absorption minimum located at $\epsilon_{2}=-q_{2}$. Obviously these profiles are quite different from those of any bound-state systems. In order for bound-state systems to exhibit similar enhanced index of refraction without absorption, it was found that initial coherence or incoherent pumping is needed $[11,17]$. Figure 2 shows representative profiles of the linear susceptibility and its derivative for a double-autoionizing system, which is to be compared with Fig. 1. In this example we have chosen the asymmetry parameters to be $q_{2}=q_{3}=3$ and the energy separation $b \equiv E_{3}-E_{2}=3 \Gamma_{2}$. For simplicity we have also assumed that $\Gamma_{2}=\Gamma_{3}$. Note that the profiles are now similar to those for the EIT scheme. This is not surprising, since this
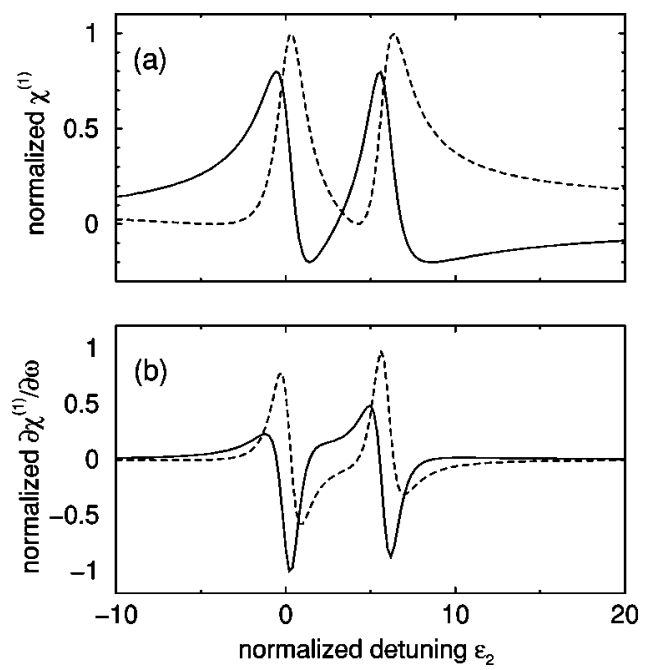

FIG. 2. (a) Real and imaginary parts of the linear susceptibility $\chi^{(1)}$ for the double-autoionizing system as a function of normalized detuning $\epsilon_{2}$. (b) Real and imaginary parts of $\partial \chi^{(1)} / \partial \omega$. Normalization is to the peak value of the imaginary part of $\chi^{(1)}$.

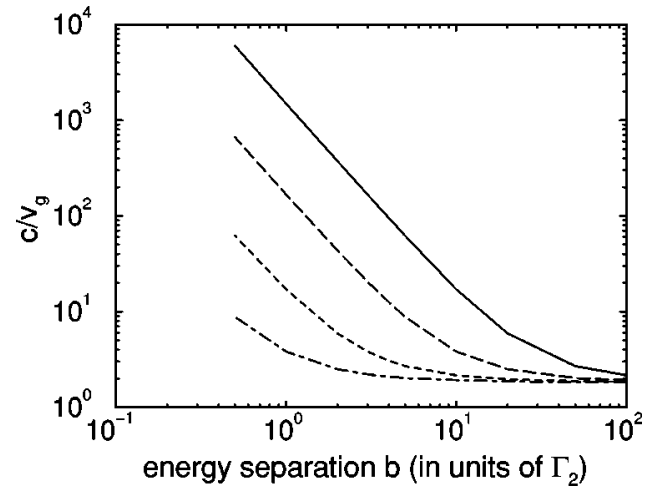

FIG. 3. Ratio of the group velocity and light speed, $c / v_{g}$, at the interference minimum of the double-autoionizing system as a function of energy separation $b$ for different asymmetry parameters $q_{2}$ $=q_{3}=30$ (solid), 10 (dashed), 3 (dotted), and 1 (dot-dashed).

double-autoionizing system in a bare-state picture resembles the EIT system in a dressed-state picture. In other words, as explained in the beginning of this paper the doubleautoionizing system is already dressed by nonradiative intraatomic interaction without a dressing laser, by which the two autoionizing states communicate via the continuum. Mathematically this is represented by $\widetilde{\Omega}_{32}$ in Eq. (10).

We now estimate the typical value of the group velocity at the absorption minimum for the double-autoionizing system. For that purpose we have considered the following situations As we have already seen in Eq. (16), what matters is the ratio of $\sigma_{0} / \Gamma_{2}$, and in reality $\sigma_{0}$ is typically in the range of 0.1 $<\sigma_{0}<10$ (in units of $\mathrm{Mb}$ ) for most of the neutral atoms. Knowing this, it is rather realistic to assume that $\sigma_{0}$ $=1(\mathrm{Mb})$ and the autoionization width is chosen to be $\Gamma_{2}$ $=1 \mathrm{~cm}^{-1}$ or $10 \mathrm{~cm}^{-1}$, since autoionization width of neutral elements typically ranges from a fraction of $1 \mathrm{~cm}^{-1}$ to a few hundred $\mathrm{cm}^{-1}$. Atomic density is chosen to be $N$ $=10^{18} \mathrm{~cm}^{-3}$. For simplicity we further assume that $\Gamma_{3}$ $=\Gamma_{2}$ and the transition wavelength $\lambda=100 \mathrm{~nm}$. A simple estimation shows that the collisional and Doppler widths for metal elements are typically less than $0.25 \mathrm{~cm}^{-1}$ even at the density of $10^{18} \mathrm{~cm}^{-3}$ and the temperature of $1200 \mathrm{~K}$ or so, justifying our choice of atomic density.

In Fig. 3 we show the variation of the group velocity as a function of energy separation $b$ for different values of asymmetry parameters $q_{2}$ and $q_{3}$. As $b \rightarrow \infty$, the group velocity becomes independent of the values of $q_{2}$ and $q_{3}$, which means that the system essentially approaches an isolatedautoionizing system, whose group velocity is independent of asymmetry parameter once $\sigma_{0}$ and $\Gamma_{2}$ are given, as we have already seen in Eq. (16). On the other hand, as $b \rightarrow 0$, the reduction of the group velocity is more prominent when asymmetry parameters are larger.

Finally in Fig. 4 we present the dependence of the group velocity on the ratio of $\sigma_{0} / \Gamma_{2}$ as a function of energy separation $b$. Roughly speaking, the group velocity becomes one order of magnitude larger (smaller) as the ratio $\sigma_{0} / \Gamma_{2}$ becomes one order of magnitude larger (smaller). A similar tendency has been found for different values of asymmetry parameters (not shown here). 


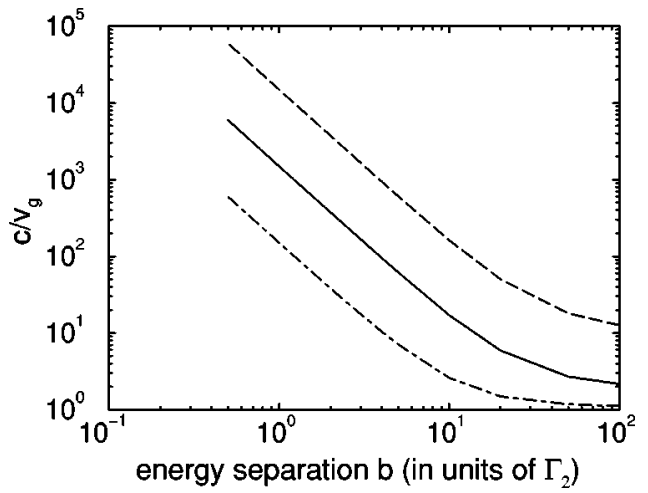

FIG. 4. Ratio of the group velocity and light speed, $c / v_{g}$, at the interference minimum of the double-autoionizing system as a function of energy separation $b$ for different ratios of $\sigma_{0} / \Gamma_{2}=10$ (dashed), 1 (solid), and 0.1 (dot-dashed). $q_{2}=q_{3}=30$ for all curves.

\section{CONCLUSIONS}

In conclusion we have studied the linear and nonlinear optical properties of autoionizing systems under the action of a single-laser field. When the system involves an isolatedautoionizing state, we have found that the group velocity at the absorption minimum is independent of the value of the asymmetry parameter for a given ionization cross section into the smooth continuum $\sigma_{0}$ and an autoionization width $\Gamma_{2}$. Under typical situations the reduction of the group velocity is rather small and may not be larger than 100. However, the reduction of the group velocity can be significant by the presence of a neighboring-autoionizing state when the laser frequency is tuned to the interference minimum between two autoionizing states. It should be noted that the effect of the presence of the neighboring-autoionizing state is more pronounced if the asymmetry parameters take larger values. This is because the interference minimum of the double-autoionizing system becomes much steeper than that of the isolated-autoionizing system, and the steepness increases as the values of asymmetry parameters increase. Although the reduction of the group velocity for our scheme is not as striking as that for the EIT scheme, it may be still useful, especially in the VUV-XUV regime, since there are less experimental requirements in terms of laser bandwidths, intensities, and atomic density, etc., for experimental realization.

\section{ACKNOWLEDGMENT}

This work was supported by the Grant-in-Aid for scientific research from the Ministry of Education, Japan.
[1] K.-J. Boller, A. Imamoğlu, and S.E. Harris, Phys. Rev. Lett. 66, 2593 (1991).

[2] S.E. Harris, Phys. Rev. Lett. 62, 1033 (1989).

[3] S.E. Harris, J.E. Field, and A. Kasapi, Phys. Rev. A 46, R29 (1992).

[4] A. Kasapi, M. Jain, G.Y. Yin, and S.E. Harris, Phys. Rev. Lett. 74, 2447 (1995)

[5] L.V. Hau, S.E. Harris, Z. Dutton, and C.H. Behroozi, Nature (London) 397, 594 (1999).

[6] M.M. Kash, V.A. Sautenkov, A.S. Zibrov, L. Hollberg, G.R. Welch, M.D. Lukin, Y. Rostovtsev, E.S. Fry, and M.O. Scully, Phys. Rev. Lett. 82, 5229 (1999).

[7] K.H. Hahn, D.A. King, and S.E. Harris, Phys. Rev. Lett. 65, 2777 (1990).
[8] S.E. Harris, J.E. Field, and A. Imamoğlu, Phys. Rev. Lett. 64, 1107 (1990).

[9] H. Schmidt and A. Imamoğlu, Opt. Lett. 21, 1936 (1996).

[10] Takashi Nakajima, Opt. Lett. 25, 847 (2000).

[11] M. Fleischhauer, C.H. Keitel, M.O. Scully, Chang Su, B.T. Ulrich, and Shi-Yao Zhu, Phys. Rev. A 46, 1468 (1992).

[12] U. Rathe, M. Fleischhauer, Shi-Yao Zhu, T.W. Hänsch, and M.O. Scully, Phys. Rev. A 47, 4994 (1993).

[13] A. Lyras, X. Tang, P. Lambropoulos, and Jian Zhang, Phys. Rev. A 40, R4131 (1989).

[14] S.-Y. Zhu and E.E. Fill, Phys. Rev. A 42, 5684 (1990).

[15] P. Lambropoulos and P. Zoller, Phys. Rev. A 24, 379 (1981).

[16] Takashi Nakajima, Phys. Rev. A 60, 4805 (1999).

[17] M.O. Scully, Phys. Rev. Lett. 67, 1855 (1991). 